\title{
Plasmon-induced Trap State Emission from Single Quantum Dots
}

\author{
Junyang Huang ${ }^{1}$, Oluwafemi S Ojambati ${ }^{1}$, Rohit Chikkaraddy ${ }^{1}$, Kamil Sokołowski ${ }^{2}$, Qifang Wan ${ }^{3}$, Colm \\ Durkan ${ }^{3}$, Oren A Scherman ${ }^{2}$, Jeremy J Baumberg ${ }^{1, *}$ \\ ${ }^{1}$ NanoPhotonics Centre, Cavendish Laboratory, Department of Physics, University of Cambridge, CB3 OHE, UK \\ ${ }^{2}$ Melville Laboratory for Polymer Synthesis, Department of Chemistry, University of Cambridge, CB2 1EW, UK \\ ${ }^{3}$ Nanoscience Center, University of Cambridge, $11 \mathrm{JJ}$ Thomson Avenue, Cambridge CB3 OFF, UK
}

Charge carriers trapped at localised surface defects play a crucial role in quantum dot (QD) photophysics. Surface traps offer longer lifetimes than band-edge emission, expanding the potential of QDs as nanoscale light-emitting excitons and qubits. Here, we demonstrate that a non-radiative plasmon mode drives the transfer from two-photon-excited excitons to trap states. In plasmonic cavities, trap emission dominates while the band-edge recombination is completely suppressed. The induced pathways for excitonic recombination not only shed light on the fundamental interactions of excitonic spins, but also open new avenues in manipulating QD emission, for optoelectronics and nanophotonics applications.

Quantum dots (QDs) find extensive applications in optoelectronic devices [1,2], (photo)catalysis [3], and biosensing.[4] Improving their exciton recombination is critical for utilisation, and marrying QDs with optical cavities is promising for tuning their photonic properties. Single photon sources, $[5,6]$ stronglycoupled light-matter polaritons [7-11], and nonlinear optical control [12] embed QDs inside various optical nanocavities. Controlling hybridisation of quantum dots with other nanophotonic components is thus crucial. Chemically synthesised colloidal quantum dots provide unparalleled simplicity of scaleup fabrication and straightforward integration into nano-architectures down to the single emitter level. [5,7-9,13-15] However their properties strongly depend on surface chemical composition and structure which have been hard to control.

The QD surface impacts their optoelectronic properties. The lower coordination of QD surface atoms and a variety of ligand binding modes lead to a distribution of localised charge trapping sites. [16] These trap sites reduce photoluminescence (PL) quantum efficiency [17] and constrain applications in energy conversion. [18] Such surface traps can be suppressed by chemical etching $[19,20]$, surfacebound ligands [21,22], and passivation with widebandgap shells [23], however, complete elimination is hindered due to incomplete ligand coordination, remaining lattice vacancies and lattice mismatch at the core-shell interface. Radiative recombination of surface-trap excitons gives long-lifetime emission redshifted from the band-edge exciton PL. For InP/ZnS core-shell colloidal QDs used here, the energy separation between the surface states and band-edge emission is $\sim 250 \mathrm{meV}$, while the band-edge bright/ dark exciton splitting is $\sim 10 \mathrm{meV}$, allowing them to be distinguished. [24] The large surface:volume ratio of QDs allows dense surface traps to emit as brightly as the band-edge excitons, giving 'white light' QD emission. $[22,25,26]$

While trap emission was initially attributed to surface indium dangling bonds, [27] more recent EPR [28] and XPS studies of HF-etched InP QDs evidence the role of hole-trapping $\mathrm{P}$ dangling bonds and surface oxidation. Ab-initio calculations [29], positron spectroscopy [30], XPS [31], and optical magnetic resonance [32] of CdSe QDs also show that surface traps stem from dangling selenium bonds (analogous effects found also for CdS QDs [33]). Despite this wealth of information, there is less understanding of their influence in optical cavities, as well as the interplay with intrinsic excitons. [34]

Here, we demonstrate for the first time that the carrier recombination process in QDs is significantly modified by a plasmonic nanocavity, resulting in dominant trap state emission under two-photon excitation. We use a 'nanoparticle-on-mirror' (NPoM) geometry [35], built of individual Au nanoparticles above a Au film with QDs sandwiched between the metallic surfaces (Fig. 1a) to provide an optical field enhancement up to $E / E_{0}=100$ in a mode volume $<100 \mathrm{~nm}^{3}$. This deep sub- $\lambda$ extreme confinement of light in NPoMs enhances two-photon absorption $>10^{8}$, switching on nonlinear interactions [36] and manipulating absorption/emission in single QDs. [37]

Despite studies of surface traps using one-photon excitation, interactions between spin-forbidden dark excitons and surface traps remains largely unknown. Quantum confinement modifies the QD excitonic states, each of specific parity. Dark exciton states have odd parity, thus one-photon interband transitions to these states are forbidden. [38-42] Resonant absorption to excitonic dark states can however be achieved using two-photon excitation. [40-43] 

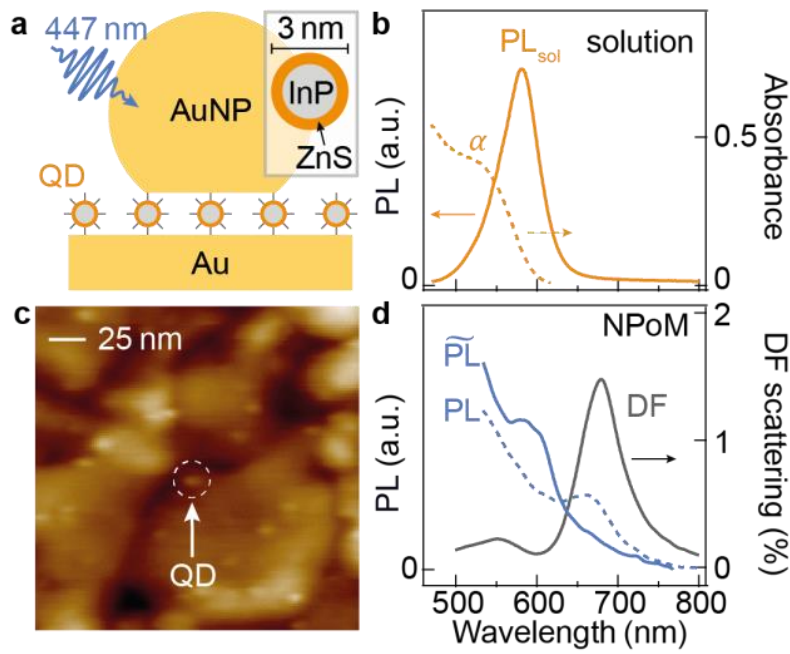

FIG. 1. (a) Schematic of QDs in NPoM nanogap. Au NP sits on monolayer of quantum dots self-assembled onto gold film, inset shows core-shell InP/ZnS QDs. (b) Photoluminescence spectrum of InP/ZnS QD $540\left(\lambda_{\max }=540 \mathrm{~nm}\right)$ in solution (solid line), along with extinction spectrum (dashed line). (c) AFM image of QDs on gold substrate. (d) PL spectra of QDs coupled to the plasmonic cavity (dashed), dark-field spectrum of the NPoM cavity (grey), and QD emission spectrum normalised by the cavity resonance (solid blue).

The three types of $\operatorname{InP} / \mathrm{ZnS}$ core-shell QDs with exciton absorption at $460 \mathrm{~nm}, 540 \mathrm{~nm}, 580 \mathrm{~nm}\left(\mathrm{QD}_{460}, \mathrm{QD}_{540}\right.$, $\mathrm{QD}_{580}$ ) used here (see SI Note 1) are stabilised with 3mercaptopropionate ligands (MPA) to sustain their dispersion in water and allow close proximity to $\mathrm{Au}$. [44] The $\mathrm{QD}_{540}(3.1 \pm 0.3 \mathrm{~nm})$ solution exhibits an exciton absorption edge at $540 \mathrm{~nm}$ while their room temperature photoluminescence $(\mathrm{PL})$ is centred at $580 \mathrm{~nm}$ for $447 \mathrm{~nm}$ excitation (Fig. 1b). We immerse template-stripped ultraflat $\mathrm{Au}$ substrates in the $\mathrm{QD}$ solution, self-assembling QDs onto the $A u$ film through the binding of the MPA ligands to the Au surface. [45] Atomic force microscopy (AFM) reveals a sparse distribution of separated individual QDs with a surface coverage of $\sim 2 \times 10^{-4}$ QDs nm ${ }^{-2}$ (Fig. 1c). This implies that when drop-casting $80 \mathrm{~nm} \mathrm{Au}$ nanoparticles on top, only a minority of assembled NPoM cavities contain more than a single QD $(<1 \%$, SI Note 3$)$. Modelling the QD-filled NPoM cavity using finite-difference time-domain calculations gives maximum field enhance-ment $E / E_{0}=100$ (Fig. S7). The resulting Purcell effect drastically reduces the emission lifetime of QDs embedded in NPoM cavities. [46] The predicted $\tau<100 \mathrm{fs}$ is well below any single-photon avalanche diode instrument response (>50 ps, Fig. S8), hindering confirmation of singlephoton generation.

With one-photon excitation at $447 \mathrm{~nm}, \mathrm{PL}$ of QDs on flat $\mathrm{Au}$ is strongly quenched due to non-radiative coupling into the metal (Fig. S9a). [46] By contrast, PL of the QDs recovers when embedded within the
NPoM cavity as the radiative decay rate is now Purcell-enhanced and competes with non-radiative relaxation. $[48,49]$ The PL spectral shape is strongly modified (dashed blue curve, Fig. 1d) as the QD emission couples with the gap plasmon modes of the NPoM. The observed emission peak arises from the lowest coupled plasmon resonance, confirmed by its red-shifting with the NPoM dark-field scattering peak when the facet of the Au nanoparticle restructures over time (Fig. S9c,d). Normalizing the measured PL spectrum to the outcoupling efficiency of the same NPoM structure, which is given by the square root of the dark-field scattering spectrum [36] (grey curve, Fig. 1d), yields the true emission peak $(\widetilde{P L})$ around $590 \mathrm{~nm}$ (blue curve, Fig. 1d), matching the emission peak measured from QDs on glass. The additional shorter-wavelength background is light emission from the metal, which is strongest near the bulk plasmon at 510nm. [50]
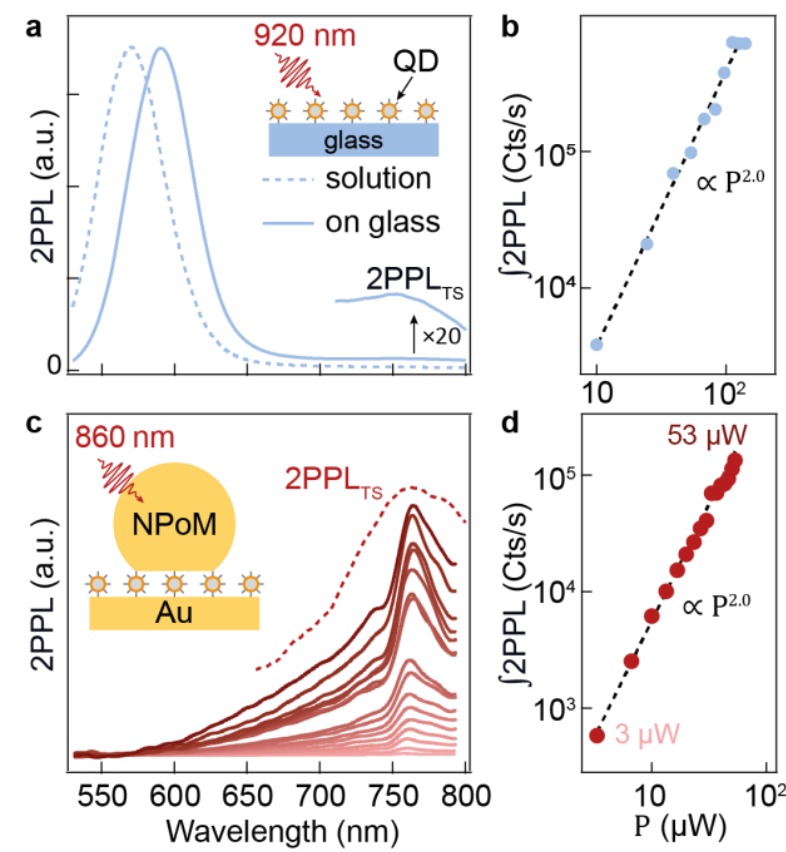

FIG. 2. Two-photon excited photoluminescence (2PPL). (a) $Q_{540}$ emission spectrum excited by $120 \mathrm{fs}$ pulses at $920 \mathrm{~nm}$, for QDs in aqueous phase/air (dashed line) and dried on glass (solid line, as in inset). (b) Power dependence of 2PPL signals from $Q_{540}$ solution, confirming emission is from two-photon absorption. (c) Emission spectra of QDs in a NPoM cavity (as in inset) for increasing excitation power (solid line). Also shown (dashed line) is two-photonabsorption trap-state emission from QDs dried on glass. (d) Quadratic power dependence of QD PL in NPoM.

We probe the exciton level structure in $\mathrm{InP} / \mathrm{ZnS}$ QDs using two-photon excitation (2PPL). With $120 \mathrm{fs}$ pulses at $920 \mathrm{~nm}$, both QDs in aerobic aqueous solution and QDs dried on glass (SI Note 2) show bandedge emission similar to their 1PPL spectra (Figs. 2a, S10a). The quadratic dependence of the integrated emission intensity on the average excitation power (Fig. 2b) confirms that the emission 
comes from nonlinear two-photon absorption (2PA). For 2PA in QDs dried on glass, trap state emission is 40 times weaker than the band-edge emission. Control experiments with 1PPL indeed verify this longwavelength emission is from trap states (Fig. S10a).

In contrast, for QDs coupled to the NPoM cavity, two-photon excitation induces drastically different emission spectra with a broad peak at $760 \mathrm{~nm}$, while the band-edge emission is completely suppressed (Fig. 2c). This emission is again quadratic with power (Fig. 2d) and consistent from $>50$ NPoM nanocavities examined (Fig. S11), yet absent from NPoM samples without QDs. The spectral shape and resonance position of the NPoM 2PPL emission coincide with the 2PA trap state emission from QDs on glass (Fig. 2c,

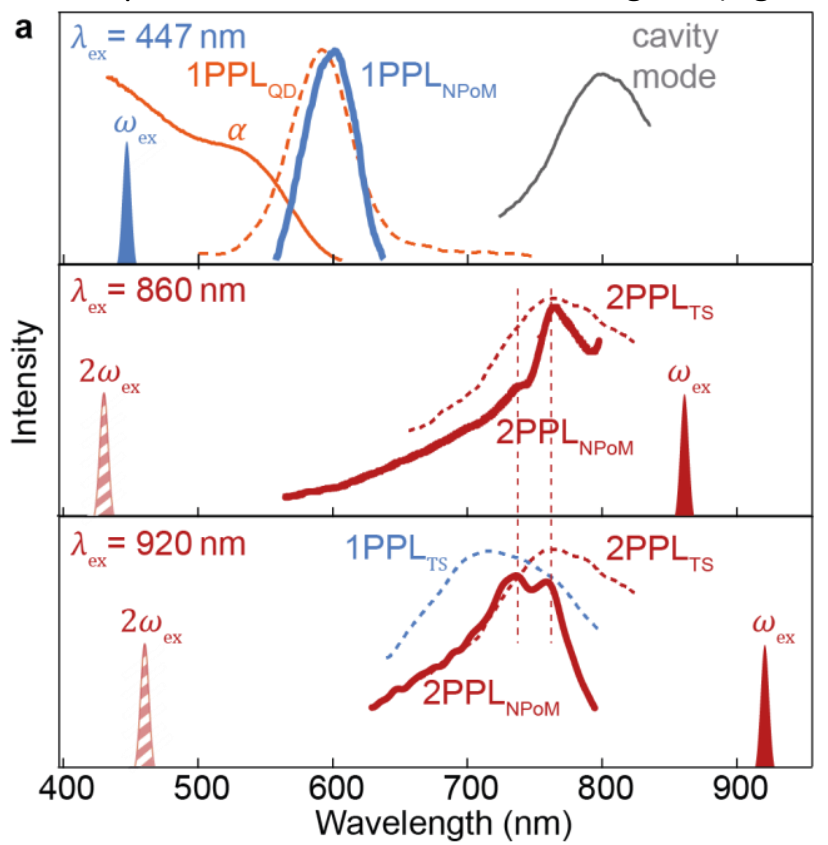

dashed line). This confirms that the excitonic recombination process of the QD is strongly modified by the NPoM cavity so that the dominant emission originates now from surface trap states.

To understand the exciton relaxation and emission from QDs in NPoM cavities, we compare emission spectra with different excitation wavelengths. For one-photon interband excitation at $447 \mathrm{~nm}$, we observe dominant emission from the band-edge bright exciton (Fig. 3a, top panel). With two-photon excitation at $860-920 \mathrm{~nm}$, the band-edge emission is completely turned off while broad trap state emission (FWHM $\sim 100 \mathrm{~nm}$ ) is enhanced (Fig. 3a, middle and bottom panels).
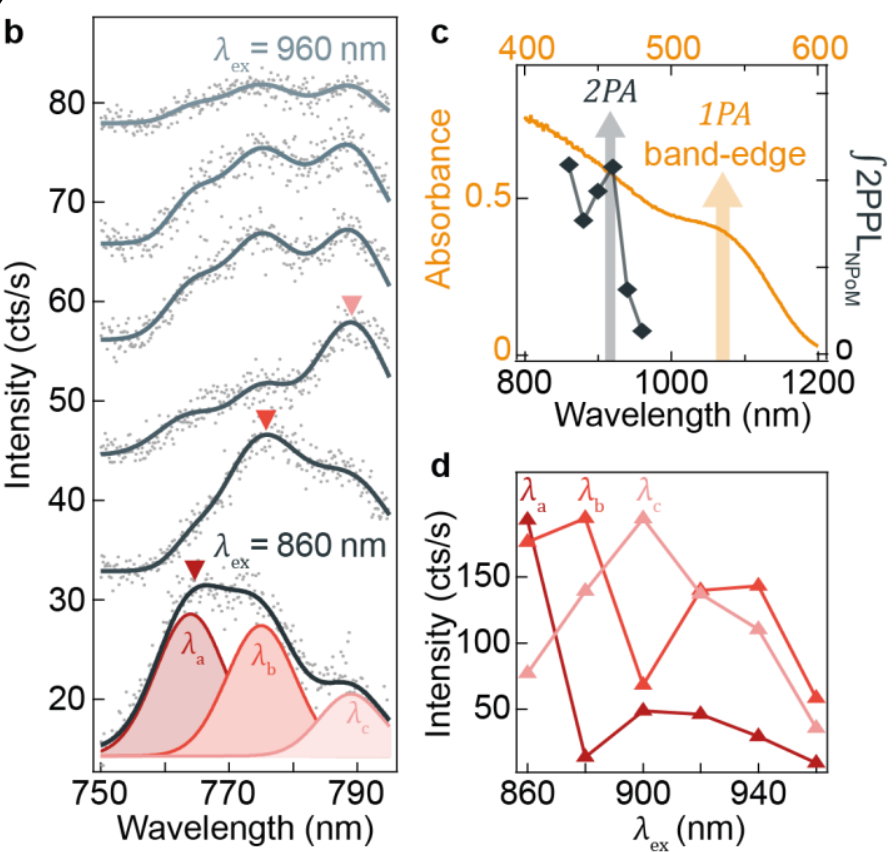

FIG. 3. Selectively-pumped trap state emission. (a) Top panel: Normalised PL from one-photon-absorption of QDs in NPoM cavity ( $\widetilde{P L}$, blue line) and on glass (dashed orange line) using $\mathrm{CW}$ excitation at $447 \mathrm{~nm}$, along with extinction of QDs in solution (solid orange line) and plasmonic cavity resonance (grey line). Middle/bottom panels: 2PPL spectrum for QDs in NPoM (solid line) with (middle) $860 \mathrm{~nm}$ and (bottom) $920 \mathrm{~nm}$ pulsed excitation, and from QDs dried on glass (red dashed, while blue dashed line shows from one-photon excitation). Excitation wavelengths and equivalent one-photon doubled wavelengths also marked. (b) 2PPL spectra for a single QD NPoM vs pump excitation tuned from $860 \mathrm{~nm}$ to 960 $\mathrm{nm}$ (20 nm steps), with fit curves (grey line) composed of three Gaussians at 764, 775, and $789 \mathrm{~nm}$ (red arrows). (c) Integrated 2PPL intensity of QDs in NPoM vs excitation wavelength showing absorption resonance $\sim 920 \mathrm{~nm}$ (grey line) and one-photon $\alpha$ spectrum of QD solution plotted $v s \lambda / 2$ axis (yellow line). (d) Integrated intensity for the three 2PPL modes vs excitation wavelength, showing selective pumping of individual QD trap states.

Surprisingly, different excitation wavelengths $\lambda_{\text {ex }}$ selectively populate different trap states. With $\lambda_{\text {ex }}=860 \mathrm{~nm}$, trap state emission is peaked $\sim 760 \mathrm{~nm}$, while using $\lambda_{\mathrm{ex}}=920 \mathrm{~nm}$ yields an additional 2PPL peak at $730 \mathrm{~nm}$ (Fig. 3a, bottom panel) that is consistently observed across 30 NPoM constructs examined. These large changes in $2 \mathrm{PPL}$ provide insight into the discrete energy levels within the trap state and their selective coupling to the excitons excited by 2PA inside nanocavities. Such discrete energy states cannot be distinguished in the broad trap state 2PPL band acquired from QDs outside cavities (Fig. 2a).

To gain more understanding of this trap state emission, we collect the 2PPL signal while scanning $\lambda_{\text {ex }}$ from $860 \mathrm{~nm}$ to $960 \mathrm{~nm}$ on each QD-filled NPoM (Fig. 3b). The integrated trap state emission within our detection window is found to peak for pump $\lambda_{\mathrm{ex}}=920 \mathrm{~nm}$, corresponding to a dark $(J=2)$ excitonic absorption resonance at $460 \mathrm{~nm}$ (Fig. 3c). The $J=2$ exciton is thus $0.35 \mathrm{eV}$ higher energy than the $J=1$ 
ground state, suggesting that it arises from the $p$ state manifold of the $Q D$, as discussed below.

The 2PPL spectra when tuning $\lambda_{\mathrm{ex}}$ are all composed of the same set of emission modes at $\lambda_{\mathrm{a}}=764 \mathrm{~nm}, \lambda_{\mathrm{b}}=775 \mathrm{~nm}$, and $\lambda_{\mathrm{c}}=789 \mathrm{~nm}$ (Fig. S12). For $\lambda_{\text {ex }}=860 \mathrm{~nm}$ strongest emission comes from the two $\lambda_{\mathrm{a}, \mathrm{b}}$ higher energy modes, while tuning to $\lambda_{\mathrm{ex}}=880 \mathrm{~nm}$ elicits redder emission dominated by $\lambda_{\mathrm{b}, \mathrm{c}}$ (Fig. 3d). Further increasing $\lambda_{\mathrm{ex}}$ to $900 \mathrm{~nm}$ hits the resonance of the lowest energy trap $\left(\lambda_{c}\right)$. Not only does lower energy excitation selectively induce emission from lower energy trap states, but the energy difference ( $\left.\Delta E=2 \omega_{\mathrm{ex}}-E_{\mathrm{TS}}\right)$ between the trap state emission $\left(E_{\mathrm{TS}}\right)$ and two-photon absorption energy remains fixed at $\Delta E \simeq 1.2 \mathrm{eV}$. This implies energetic relaxation is dominated by a single resonant process from the $J=2$ exciton to the trap states. The contrast between 2PPL spectra of QDs inor outside NPoMs implies preferential exciton relaxation into trap states for plasmonic cavities. To investigate further, the energies of band-edge and trap states are tuned by varying the QD size. The onephoton excited band-edge emission redshifts as expected for reduced electron confinement in the larger $Q_{580}$ (Fig. 4a). Similarly, larger QDs redshift the trap state emission band with two-photon excitation (Fig. 4b dashed), with slight drop of energy separation $E_{\mathrm{g}}-E_{\mathrm{TS}}$ from 0.7 to $0.5 \mathrm{eV}$ (Fig. S13). In these solution QDs, two-photon-excited dark excitonic states undergo rapid non-radiative phonon relaxation from the $J=2$ exciton to the exciton ground state $\left(E_{\mathrm{g}}\right)$ through phonon emission. [51] At room temperature, the band-edge bright and dark excitons are thermally mixed, leading to PL from the bright exciton recombination (Fig. 4c). This process is also accompanied by exciton relaxation into surface trap states $\left(E_{\mathrm{TS}}\right)$, which gives weak emission of a lower energy photon upon recombination.

Using NPoMs with QDs of increasing size shows the 2PPL emission spectra redshifting exactly as for the 2PPL from solution QDs (Fig. $4 \mathrm{~b}$ solid lines). This implies that indeed it is the relaxation rates rather than the electronic states themselves which change in the plasmonic cavity, and that the suppression of band-edge exciton emission is universal. We note that the plasmon cavity mode also redshifts for larger QDs (Fig. 4d), due to the larger gap refractive index [52] (Fig. S14a).

The dominance of trap state emission for $J=2$ excitation is unexpected. Our data reveal that an ultrafast ( $<500 \mathrm{fs}$ ) [53] spin-preserving transition of $\Delta E \sim 1.2 \mathrm{eV}$ is responsible, and is produced by plasmonic confinement. It is suggestive that this $\Delta E$ is close to the dark-plasmon mode denoted (11) that lies below the bright coupled plasmon visible in Fig. 4 d. One possibility is thus that $J=2$ excitons rapidly emit (11) gap plasmons (which since also dark are mostly absorbed in the metal) to transfer to $J=1$ trap states. For two-photon excitation, QDs in
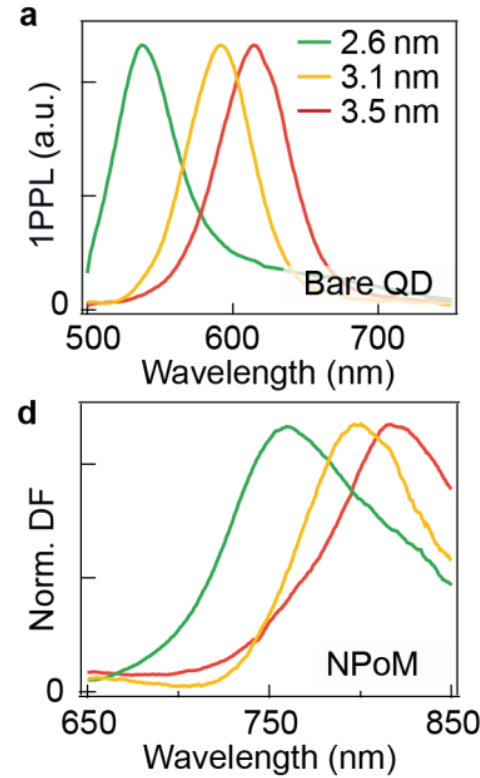
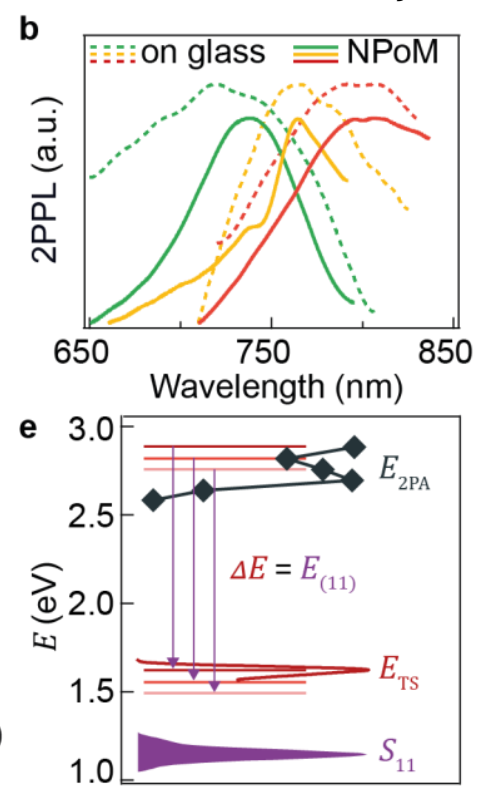

C
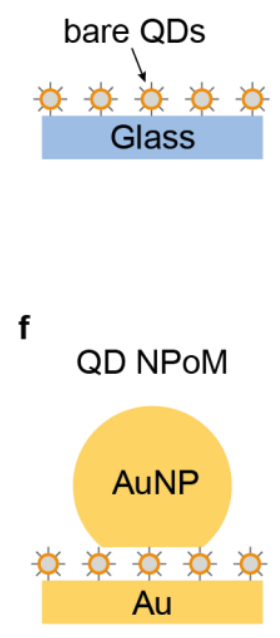
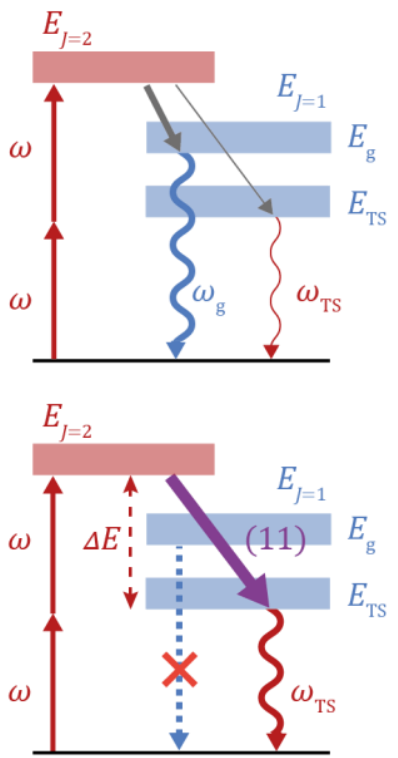

FIG. 4. NPoM-modified radiative recombination processes. (a) Ensemble PL from one-photon-absorption of $Q D_{460,540,580}$ on glass. (b) Trap state PL from TPA of QDs1-3 on glass (dashed) and inside NPoM cavities (solid line), using $920 \mathrm{~nm}$ pulses. (c) Bare QDs on glass: two-photon excitation of dark $J=2$ exciton (double red arrows). Excitons either spin-flip to the ground state $E_{g}$ (grey arrow) to emit PL (blue arrow), or couple to $J=2$ surface traps to emit red-shifted photons. (d) Darkfield spectra of NPoM cavities formed with QDs1-3. (e) Energy alignment of $J=2$ excitons ( $E_{2 \mathrm{PA}}$, black points show 2PA), emissive surface traps $\left(E_{\mathrm{TS}}\right)$, and calculated (11) plasmon mode of NPoM. (f) Model of QDs in NPoM: two-photonexcited dark exciton relaxed by (11) gap plasmon into surface trap states, bypassing formation of band-edge excitons. 
NPoMs are found to emit trap state light $>4000$ more brightly than the band-edge emission from QDs in solution, confirming highly radiative coupling is possible from such $J=1$ trap states.

To support this hypothesis, we perform finitedifference time-domain simulations to reveal both bright and dark electromagnetic modes of the NPoM plasmonic cavity (Fig. S14a,b). These identify the non-radiative 'in-plane' plasmon mode (11) $[54,55]$ at $1.1 \mathrm{eV}$, which persists under variations of facet width and nanoparticle size (Fig. S15). This (11) energy matches the relaxation energy $\Delta E$ (Fig. 4e) supporting our hypothesis of plasmon-induced excitonic relaxation. This process is forbidden from the band-gap excitons both energetically and by spin (since the resulting $J=2$ trap states would not radiate). In addition, we demonstrate that such plasmoninduced trap state emission is also observed with CdTe QDs in NPoM cavities (Fig. S16).

The QDs in these nanogaps are expected to have random lattice orientations with respect to the NPoM facet. Although the optical field orientation in the NPoM cavity is perpendicular to the facet, the excited excitons confined in each QD have non-zero in-plane components that can couple to in-plane dipolar plasmon modes. Because the NPoM nanocavity mode around $800 \mathrm{~nm}$ has perpendicular field, it is not possible to use it to inject specific spin states $\left(J_{z}= \pm 1\right)$ using circular polarisation. However it does appear from our data that the bright and dark plasmonic modes can considerably mix and influence the excitonic recombination process in QD NPoM cavities.

In conclusion, we experimentally reveal that NPoM plasmonic nanocavities strongly modify the emission of single QDs by fully suppressing the bandedge emission and enhancing two-photon-excited trap state emission. We resolve a number of different excitonic trap states $0.5-0.7 \mathrm{eV}$ below the $J=1$ exciton which are $15-75 \mathrm{meV}$ apart (Fig. S12), possibly related to hole-band mixing at defects. These effects persist for different QD sizes, which also tune the trap state emission wavelength. We explain our observations by a plasmon-induced ultrafast decay from the $J=2$ excitons to $J=1$ trap states at resonant energy difference $\Delta E \sim 1.2 \mathrm{eV}$. We suggest that this is provided by Purcell-enhancement of a dark plasmon mode that exists in the nanogap, emitted in times $(<100 \mathrm{fs}[5,47])$ much shorter than excitonic spin-flip relaxation from the excited state to the band edge [53]. Band-edge emission would only become visible if the trap states are saturated (and higher peak powers would damage these nano-constructs).
The dressing of QDs by plasmons thus has a profound effect on their energy relaxation, as well as their emission rates (which we also observe to be Purcellenhanced in nanogaps for one-photon excitation, though note that strong coupling is not achieved here). Such tightly-confined optical fields in plasmonic cavities open challenging domains for quantum electrodynamics of quantum dots including dark excitons and surface trap excitons, and for future generations of nanophotonic devices such as high repetition rate on-chip communication and quantum information processing.

We acknowledge financial support from UK EPSRC Grants EP/L027151/1, EU THOR 829067 and POSEIDON 861950. J.H. acknowledges support from Cambridge Trust and CSC scholarship. O.S.O. acknowledges a Rubicon fellowship of Netherlands Organisation for Scientific Research. R.C. acknowledges support of Trinity College Cambridge. K.S acknowledges European Commission for Marie Curie Fellowships (ESTIMABLeNANO, no. 706425).

\section{REFERENCES}

[1] I. Aharonovich, D. Englund, and M. Toth, SolidState Single-Photon Emitters, Nature Photon 10, 631 (2016).

[2] M. V. Kovalenko, L. Protesescu, and M. I. Bodnarchuk, Properties and Potential Optoelectronic Applications of Lead Halide Perovskite Nanocrystals, Science 358, 745 (2017).

[3] M. A. Boles, M. Engel, and D. V. Talapin, SelfAssembly of Colloidal Nanocrystals: From Intricate Structures to Functional Materials, Chem. Rev. 116, 11220 (2016).

[4] J. Zhou, Y. Yang, and C. Zhang, Toward Biocompatible Semiconductor Quantum Dots: From Biosynthesis and Bioconjugation to Biomedical Application, Chem. Rev. 115, 11669 (2015).

[5] T. B. Hoang, G. M. Akselrod, and M. H. Mikkelsen, Ultrafast Room-Temperature Single Photon Emission from Quantum Dots Coupled to Plasmonic Nanocavities, Nano Lett. 16, 270 (2016).

[6] A. Reinhard, T. Volz, M. Winger, A. Badolato, K. J. Hennessy, E. L. Hu, and A. Imamoğlu, Strongly Correlated Photons on a Chip, Nature Photonics 6, 2 (2012).

[7] H. Leng, B. Szychowski, M.-C. Daniel, and M. Pelton, Strong Coupling and Induced Transparency at Room Temperature with Single Quantum Dots and Gap Plasmons, Nat Commun 9, 4012 (2018). 
[8] H. Groß, J. M. Hamm, T. Tufarelli, O. Hess, and B. Hecht, Near-Field Strong Coupling of Single Quantum Dots, Science Advances 4, eaar4906 (2018).

[9] K. Santhosh, O. Bitton, L. Chuntonov, and G. Haran, Vacuum Rabi Splitting in a Plasmonic Cavity at the Single Quantum Emitter Limit, Nature Communications 7, 1 (2016).

[10] K. Srinivasan and O. Painter, Linear and Nonlinear Optical Spectroscopy of a Strongly Coupled Microdisk-Quantum Dot System, Nature 450, 7171 (2007).

[11] J. M. Katzen, C. Tserkezis, Q. Cai, L. H. Li, J. M. Kim, G. Lee, G.-R. Yi, W. R. Hendren, E. J. G. Santos, R. M. Bowman, and F. Huang, Strong Coupling of Carbon Quantum Dots in Plasmonic Nanocavities, ACS Appl. Mater. Interfaces 12, 19866 (2020).

[12] T. Volz, A. Reinhard, M. Winger, A. Badolato, K. J. Hennessy, E. L. Hu, and A. Imamoğlu, Ultrafast All-Optical Switching by Single Photons, Nature Photonics 6, 9 (2012).

[13] R. A. Jensen, I.-C. Huang, O. Chen, J. T. Choy, T. S. Bischof, M. Lončar, and M. G. Bawendi, Optical Trapping and Two-Photon Excitation of Colloidal Quantum Dots Using Bowtie Apertures, ACS Photonics 3, 423 (2016).

[14] I. Fushman, D. Englund, A. Faraon, N. Stoltz, P. Petroff, and J. Vučković, Controlled Phase Shifts with a Single Quantum Dot, Science 320, 769 (2008).

[15] R. Yalla, M. Sadgrove, K. P. Nayak, and K. Hakuta, Cavity Quantum Electrodynamics on a Nanofiber Using a Composite Photonic Crystal Cavity, Phys. Rev. Lett. 113, 143601 (2014).

[16] H. Zhang, B. Gilbert, F. Huang, and J. F. Banfield, Water-Driven Structure Transformation in Nanoparticles at Room Temperature, Nature 424, 1025 (2003).

[17] M. Jones, S. S. Lo, and G. D. Scholes, Quantitative Modeling of the Role of Surface Traps in CdSe/CdS/ZnS Nanocrystal Photoluminescence Decay Dynamics, PNAS 106, 3011 (2009).

[18] W. U. Huynh, J. J. Dittmer, and A. P. Alivisatos, Hybrid Nanorod-Polymer Solar Cells, Science 295, 2425 (2002).

[19] S. Adam, D. V. Talapin, H. Borchert, A. Lobo, C. McGinley, A. R. B. de Castro, M. Haase, H. Weller, and T. Möller, The Effect of Nanocrystal Surface Structure on the Luminescence Properties: Photoemission Study of HF-Etched InP Nanocrystals, The Journal of Chemical Physics 123, 084706 (2005).

[20] O. I. Mićić, J. Sprague, Z. Lu, and A. J. Nozik, Highly Efficient Band-edge Emission from InP
Quantum Dots, Appl. Phys. Lett. 68, 3150 (1996).

[21] H. H.-Y. Wei, C. M. Evans, B. D. Swartz, A. J. Neukirch, J. Young, O. V. Prezhdo, and T. D. Krauss, Colloidal Semiconductor Quantum Dots with Tunable Surface Composition, Nano Lett. 12, 4465 (2012).

[22] D. R. Baker and P. V. Kamat, Tuning the Emission of CdSe Quantum Dots by Controlled Trap Enhancement, Langmuir 26, 11272 (2010).

[23] S. Kim, T. Kim, M. Kang, S. K. Kwak, T. W. Yoo, L. S. Park, I. Yang, S. Hwang, J. E. Lee, S. K. Kim, and S.-W. Kim, Highly Luminescent InP/GaP/ZnS Nanocrystals and Their Application to White Light-Emitting Diodes, J. Am. Chem. Soc. 134, 3804 (2012).

[24] L. Biadala, B. Siebers, Y. Beyazit, Mickaël. D. Tessier, D. Dupont, Z. Hens, D. R. Yakovlev, and M. Bayer, Band-Edge Exciton Fine Structure and Recombination Dynamics in InP/ZnS Colloidal Nanocrystals, ACS Nano 10, 3356 (2016).

[25] S. Sapra, S. Mayilo, T. A. Klar, A. L. Rogach, and J. Feldmann, Bright White-Light Emission from Semiconductor Nanocrystals: By Chance and by Design, Advanced Materials 19, 569 (2007).

[26] M. J. Bowers, J. R. McBride, and S. J. Rosenthal, White-Light Emission from Magic-Sized Cadmium Selenide Nanocrystals, J. Am. Chem. Soc. 127, 15378 (2005).

[27] $\mathrm{H}$. Fu and A. Zunger, InP Quantum Dots: Electronic Structure, Surface Effects, and the Redshifted Emission, Phys. Rev. B 56, 1496 (1997).

[28] O. I. Mićić, A. J. Nozik, E. Lifshitz, T. Rajh, O. G. Poluektov, and M. C. Thurnauer, Electron and Hole Adducts Formed in Illuminated InP Colloidal Quantum Dots Studied by Electron Paramagnetic Resonance, J. Phys. Chem. B 106, 4390 (2002).

[29] A. Puzder, A. J. Williamson, F. Gygi, and G. Galli, Self-Healing of CdSe Nanocrystals: FirstPrinciples Calculations, Phys. Rev. Lett. 92, 217401 (2004).

[30] S. W. H. Eijt, A. (Tom) van Veen, H. Schut, P. E. Mijnarends, A. B. Denison, B. Barbiellini, and A. Bansil, Study of Colloidal Quantum-Dot Surfaces Using an Innovative Thin-Film Positron 2DACAR Method, Nature Materials 5, 1 (2006).

[31] J. E. B. Katari, V. L. Colvin, and A. P. Alivisatos, $X$-Ray Photoelectron Spectroscopy of CdSe Nanocrystals with Applications to Studies of the Nanocrystal Surface, J. Phys. Chem. 98, 4109 (1994).

[32] E. Lifshitz, A. Glozman, I. D. Litvin, and H. Porteanu, Optically Detected Magnetic Resonance Studies of the Surface/Interface 
Properties of II-VI Semiconductor Quantum Dots, J. Phys. Chem. B 104, 10449 (2000).

[33] A. Veamatahau, B. Jiang, T. Seifert, S. Makuta, K. Latham, M. Kanehara, T. Teranishi, and Y. Tachibana, Origin of Surface Trap States in CdS Quantum Dots: Relationship between Size Dependent Photoluminescence and Sulfur Vacancy Trap States, Phys. Chem. Chem. Phys. 17, 2850 (2014).

[34] A. M. Flatae, F. Tantussi, G. C. Messina, F. De Angelis, and M. Agio, Plasmon-Assisted Suppression of Surface Trap States and Enhanced Band-Edge Emission in a Bare CdTe Quantum Dot, J. Phys. Chem. Lett. 10, 2874 (2019).

[35] J. J. Baumberg, J. Aizpurua, M. H. Mikkelsen, and D. R. Smith, Extreme Nanophotonics from Ultrathin Metallic Gaps, Nat. Mater. 18, 668 (2019).

[36] O. S. Ojambati, R. Chikkaraddy, W. M. Deacon, J. Huang, D. Wright, and J. J. Baumberg, Efficient Generation of Two-Photon Excited Phosphorescence from Molecules in Plasmonic Nanocavities, Nano Lett. (2020).

[37] T. Neuman, R. Esteban, D. Casanova, F. J. García-Vidal, and J. Aizpurua, Coupling of Molecular Emitters and Plasmonic Cavities beyond the Point-Dipole Approximation, Nano Lett. 18, 2358 (2018).

[38] J. M. An, A. Franceschetti, S. V. Dudiy, and A. Zunger, The Peculiar Electronic Structure of PbSe Quantum Dots, Nano Lett. 6, 2728 (2006).

[39] B. Diaconescu, L. A. Padilha, P. Nagpal, B. S. Swartzentruber, and V. I. Klimov, Measurement of Electronic States of PbS Nanocrystal Quantum Dots Using Scanning Tunneling Spectroscopy: The Role of Parity Selection Rules in Optical Absorption, Phys. Rev. Lett. 110, 127406 (2013).

[40] B. L. Wehrenberg, C. Wang, and P. GuyotSionnest, Interband and Intraband Optical Studies of PbSe Colloidal Quantum Dots, J. Phys. Chem. B 106, 10634 (2002).

[41] J. J. Peterson, L. Huang, C. Delerue, G. Allan, and T. D. Krauss, Uncovering Forbidden Optical Transitions in PbSe Nanocrystals, Nano Lett. 7, 3827 (2007).

[42] G. Nootz, L. A. Padilha, P. D. Olszak, S. Webster, D. J. Hagan, E. W. Van Stryland, L. Levina, V. Sukhovatkin, L. Brzozowski, and E. H. Sargent, Role of Symmetry Breaking on the Optical Transitions in Lead-Salt Quantum Dots, Nano Lett. 10, 3577 (2010).

[43] Z. Ye, T. Cao, K. O'Brien, H. Zhu, X. Yin, Y. Wang, S. G. Louie, and X. Zhang, Probing Excitonic Dark States in Single-Layer Tungsten Disulphide, Nature 513, 7517 (2014).
[44] K. Sokołowski, J. Huang, T. Foldes, J. A. McCune, D. D. Xu, B. de Nijs, R. Chikkaraddy, S. M. Collins, E. Rosta, J. J. Baumberg, and O. A. Scherman, Nanoparticle Surfactants for KineticallyArrested Photoactive Assemblies to Track LightInduced Electron Transfer, (n.d.).

[45] J. T. Hugall, J. J. Baumberg, and S. Mahajan, Surface-Enhanced Raman Spectroscopy of CdSe Quantum Dots on Nanostructured Plasmonic Surfaces, Appl. Phys. Lett. 95, 141111 (2009).

[46] O. S. Ojambati, R. Chikkaraddy, W. D. Deacon, M. Horton, D. Kos, V. A. Turek, U. F. Keyser, and J. J. Baumberg, Quantum Electrodynamics at Room Temperature Coupling a Single Vibrating Molecule with a Plasmonic Nanocavity, Nature Communications 10, 1 (2019).

[47] T. B. Hoang, G. M. Akselrod, C. Argyropoulos, J. Huang, D. R. Smith, and M. H. Mikkelsen, Ultrafast Spontaneous Emission Source Using Plasmonic Nanoantennas, Nature Communications 6, 1 (2015).

[48] N. Kongsuwan, A. Demetriadou, R. Chikkaraddy, F. Benz, V. A. Turek, U. F. Keyser, J. J. Baumberg, and O. Hess, Suppressed Quenching and Strong-Coupling of PurcellEnhanced Single-Molecule Emission in Plasmonic Nanocavities, ACS Photonics 5, 186 (2018).

[49] S. Yashima, H. Sugimoto, H. Takashina, and M. Fujii, Fluorescence Enhancement and Spectral Shaping of Silicon Quantum Dot Monolayer by Plasmonic Gap Resonances, J. Phys. Chem. C 120, 28795 (2016).

[50] J. Mertens, M.-E. Kleemann, R. Chikkaraddy, P. Narang, and J. J. Baumberg, How Light is Emitted by Plasmonic Metals, Nano Lett. 17, 2568 (2017).

[51] J. T. Stewart, L. A. Padilha, W. K. Bae, W.-K. Koh, J. M. Pietryga, and V. I. Klimov, Carrier Multiplication in Quantum Dots within the Framework of Two Competing Energy Relaxation Mechanisms, J. Phys. Chem. Lett. 4, 2061 (2013).

[52] R. Chikkaraddy, X. Zheng, F. Benz, L. J. Brooks, B. de Nijs, C. Carnegie, M.-E. Kleemann, J. Mertens, R. W. Bowman, G. A. E. Vandenbosch, V. V. Moshchalkov, and J. J. Baumberg, How Ultranarrow Gap Symmetries Control Plasmonic Nanocavity Modes: From Cubes to Spheres in the Nanoparticle-on-Mirror, ACS Photonics 4, 469 (2017).

[53] R. Singh, W. Liu, J. Lim, I. Robel, and V. I. Klimov, Hot-Electron Dynamics in Quantum Dots Manipulated by Spin-Exchange Auger Interactions, Nature Nanotechnology 14, 11 (2019). 
[54] N. Kongsuwan, A. Demetriadou, M. Horton, R. Chikkaraddy, J. J. Baumberg, and O. Hess, Plasmonic Nanocavity Modes: From Near-Field to Far-Field Radiation, ACS Photonics (2020).

[55] C. Tserkezis, R. Esteban, D. O. Sigle, J. Mertens, L. O. Herrmann, J. J. Baumberg, and J. Aizpurua, Hybridization of Plasmonic Antenna and Cavity Modes: Extreme Optics of Nanoparticle-onMirror Nanogaps, Phys. Rev. A 92, 053811 (2015).

[56] C. Tserkezis, R. Esteban, D. O. Sigle, J. Mertens, L. O. Herrmann, J. J. Baumberg, and J. Aizpurua, Hybridization of Plasmonic Antenna and Cavity Modes: Extreme Optics of Nanoparticle-onMirror Nanogaps, Phys. Rev. A 92, 053811 (2015).

[56] See Supplemental Material for further details regarding sample fabrication and characterization, which include Refs. [57-62].

[57] D. V. Talapin, N. Gaponik, H. Borchert, A. L. Rogach, M. Haase, and H. Weller, Etching of Colloidal InP Nanocrystals with Fluorides: Photochemical Nature of the Process Resulting in High Photoluminescence Efficiency, J. Phys. Chem. B 106, 12659 (2002).
[58] M. Hegner, P. Wagner, and G. Semenza, Ultralarge Atomically Flat Template-Stripped Au Surfaces for Scanning Probe Microscopy, Surf. Sci. 291, 39 (1993).

[59] F. Benz, C. Tserkezis, L. O. Herrmann, B. de Nijs, A. Sanders, D. O. Sigle, L. Pukenas, S. D. Evans, J. Aizpurua, and J. J. Baumberg, Nanooptics of Molecular-Shunted Plasmonic Nanojunctions, Nano Lett. 15, 669 (2015).

[60] M. J. Horton, O. S. Ojambati, R. Chikkaraddy, W. M. Deacon, N. Kongsuwan, A. Demetriadou, O. Hess, and J. J. Baumberg, Nanoscopy through a Plasmonic Nanolens, Proc. Natl. Acad. Sci. 117, 2275 (2020).

[61] P. Han and G. Bester, Confinement Effects on the Vibrational Properties of III-V and II-VI Nanoclusters, Phys. Rev. B 85, 041306 (2012).

[62] A. R. Salmon, M.-E. Kleemann, J. Huang, W. M. Deacon, C. Carnegie, M. Kamp, B. de Nijs, A. Demetriadou, and J. J. Baumberg, LightInduced Coalescence of Plasmonic Dimers and Clusters, ACS Nano 14, 4982 (2020). 